Check for updates

Cite this: Phys. Chem. Chem. Phys., 2017, 19, 10343

Received 8th March 2017,

Accepted 24th March 2017

DOI: $10.1039 / c 7 c p 01507 k$

rsc.li/pccp

\section{The elusive silica/water interface: isolated silanols under water as revealed by vibrational sum frequency spectroscopy $\dagger$}

\author{
Laetitia Dalstein, Elizaveta Potapova and Eric Tyrode*
}

\begin{abstract}
It has been long recognized that the surface chemistry of silica, and in particular the type and relative amount of surface bound silanol groups, plays a critical role in many of the properties associated with the material, where a typical example is the discrepant adsorption behavior observed depending on the pretreatment history of the surface. However, in spite of its importance, the direct probing of specific surface silanol groups under water has been hampered by instrumental limitations. Here we make use of vibrational sum frequency spectroscopy (VSFS) to first, identify under water the $\mathrm{OH}$ stretch of isolated surface silanols, and second, explore its acid/base behavior and dependence on the surface pretreatment method. The properties of other types of silanol groups (i.e. hydrogen bonded/geminal) are also inferred from the data. The ability to directly probe these functional groups under water represents a crucial step to further improving our understanding of this widely used mineral oxide.
\end{abstract}

\section{Introduction}

The interaction of water with silicon dioxide $\left(\mathrm{SiO}_{2}\right)$, the most abundant mineral in the continental Earth crust, ${ }^{1}$ is of obvious fundamental and practical importance in a large range of geochemical, environmental, pharmaceutical, biological and catalytical processes. $^{2-5}$ In contact with water, silica surfaces typically expose siloxane ( $\mathrm{Si}-\mathrm{O}-\mathrm{Si})$, silanol $(\mathrm{Si}-\mathrm{OH})$, and silanolate $\left(\mathrm{Si}-\mathrm{O}^{-}\right)$groups, the latter resulting from the partial deprotonation of silanols at $\mathrm{pHs}$ above the isoelectric point $(\mathrm{pH} \sim 2-3){ }^{2}$ which renders the surface negatively charged under most common conditions. The relative population, speciation and distribution of these surface groups largely control and modulate the surface chemistry and associated properties of silica. ${ }^{6}$

Compared to the bulk, where silicon atoms are typically coordinated through oxygen bridges to four additional $\mathrm{Si}$ atoms $\left(\mathrm{Q}^{4}\right)$, on the surface, this number can be lower as silicon may instead bind to one $\left(\mathrm{Q}^{3}\right)$ or two $\left(\mathrm{Q}^{2}\right)$ hydroxyl groups. ${ }^{3,7,8}$ Depending on this coordination, but also on the relative lateral proximity, different kinds of silanols have been identified ${ }^{2,3,6,7}$ (see Scheme 1): (i) isolated, single or terminal $\mathrm{Si}-\mathrm{OH}\left(\mathrm{Q}^{3}\right)$ separated by at least $3.3 \AA$ from its closest silanol neighbor and consequently not capable of forming mutual hydrogen bonds; (ii) vicinal, pair of $\mathrm{Si}-\mathrm{OHs}$ (both $\mathrm{Q}^{3}$ ) sharing a

Department of Chemistry, School of Chemical Science and Engineering, KTH Royal Institute of Technology, SE-10044 Stockholm, Sweden. E-mail: tyrode@kth.se $\dagger$ Electronic supplementary information (ESI) available: Additional experimental details, Fresnel factor corrected spectra and fitting details. See DOI: 10.1039/ c7cp01507k
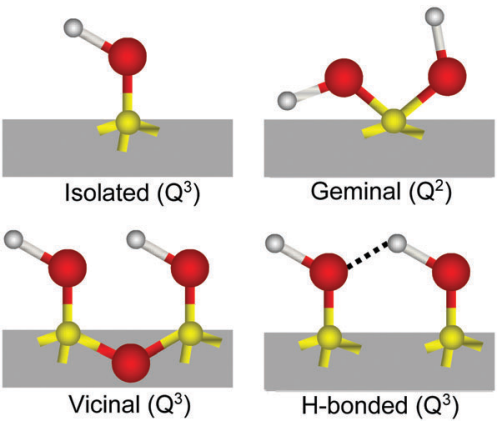

Scheme 1 Type of silanols encountered at the fused silica surface.

common oxygen vertex and thus separated by less than $3 \AA$; (iii) interacting or hydrogen bonded, $\mathrm{Q}^{3}$ silanols that are not vicinal, but nonetheless close enough to form hydrogen bonds, and finally, (iv) geminal, having two $\mathrm{OHs}$ connected to the same silicon atom $\left(\mathrm{Q}^{2}\right)$. Conclusive experimental evidence for the presence of isolated and geminal silanols has been found by IR (narrow band at $\left.\sim 3750 \mathrm{~cm}^{-1}\right)^{3,9,10}$ and NMR spectroscopy $\left({ }^{29} \mathrm{Si}\right.$ signals from $\mathrm{Q}^{2}$ silicon species), ${ }^{7,8}$ respectively. Together with the total amount of hydroxyl groups determined from deuterium exchange measurements, ${ }^{6,11}$ the relative amounts of the different types of silanols have been estimated as the surface dehydroxylates with increasing temperature. ${ }^{3,6}$ These techniques require however, large surface to volume ratios and experiments must be typically performed under vacuum or at relatively high temperatures to avoid overlapping contributions from bulk or adsorbed water molecules. 
When silica is in contact with water, the silanol $\mathrm{OH}$ signatures become largely indistinguishable from the overwhelming majority of those found in bulk $\mathrm{H}_{2} \mathrm{O}$, which limits the applicability of the above mentioned experimental approaches. Nonetheless, intrinsic surface specific techniques, such as vibrational sum frequency spectroscopy (VSFS) ${ }^{12,13}$ and second harmonic generation (SHG), ${ }^{14}$ have been able to provide information on the interfacial water in close or direct proximity to the silica surface, and in particular, upon variation of the solution $\mathrm{pH}^{15-26}$ The VSFS spectra of the silica/water interface are characterized by two broad bands centred at $\sim 3200 \mathrm{~cm}^{-1}$ and $\sim 3400 \mathrm{~cm}^{-1}$. Although the precise assignments of the two bands remain a source of debate, ${ }^{21,22,25,26}$ they are consistently linked to water vibrations in the interfacial region. Indirect information of the fate of silanols under water has been inferred by SHG, where depending on the pH history of the silica substrate, two or three successive jumps in the second harmonic response have been observed with increasing $\mathrm{pH}$. This behavior was attributed to the deprotonation of specific populations of $\mathrm{Si}-\mathrm{OH}$ having different $\mathrm{p} K_{\mathrm{a}} \mathrm{s},{ }^{17,23}$ an interpretation that has been supported by molecular dynamic simulations..$^{27-29}$ It is worth noting, however, that a large majority ( $>75 \%$ ) of surface silanols remains protonated (i.e. $\mathrm{Si}-\mathrm{OH}$ ) even at a $\mathrm{pH}$ as high as $10 .^{23,30}$ Although recent ATR-FTIR measurements on silica nanoparticles have been able to correlate a frequency shift in the Si-O stretch of surface silanols to the density of silanolate groups, ${ }^{31}$ the direct probing of specific types of SiOHs in solution remains elusive.

In the present study we have used VSFS to provide direct evidence of isolated surface silanols at the buried silica/water interface and examine some of their properties. Measurements were performed using an experimental geometry that not only significantly improves the signal to noise ratio, but also enhances the sum frequency (SF) response in the spectral region of interest. ${ }^{32}$ To facilitate the discussion of the observed features, otherwise identical fused silica hemispherical substrates were pretreated following two different procedures: plasma cleaning and heat treatment at $1000{ }^{\circ} \mathrm{C}$ in air. As will be elaborated below, we find these methods to give the largest relative variation of silanol populations at the buried interface.

\section{Experimental}

\section{Materials}

Sodium hydroxide (99.99\% trace metal basis) and hydrochloric acid (37 wt\% in water, 99.999\% trace metal basis) were obtained from Sigma Aldrich. Chromosulphuric acid, used during the cleaning procedure of the hemispheres, was from Merck. Sodium chloride (Puratronic, 99.999\% metal basis) was purchased from Alfa Aeser and then baked for at least 60 minutes at $500{ }^{\circ} \mathrm{C}$ to remove organic contaminants. Hexadecyltrimethylammonium bromide (CTAB) was obtained from Aldrich, and recrystallized three times from a $3: 1$ mixture of acetone/methanol before use. The water used in the experiments was obtained from an Integral 15 Millipore filtration unit $(18.2 \mathrm{M} \Omega \mathrm{cm}$ in resistivity, total organic carbon $<3 \mathrm{ppb}$ ). All glassware was cleaned with a commercial alkaline agent (Deconex from Borer) and rinsed thoroughly with water. The IR grade fused silica substrates (Infrasil 302) were custom-made (CVI, Melles-Griot) hemispherically shaped optics of $10 \mathrm{~mm}$ in diameter. The flat side of the hemispheres displayed a surface arithmetic average roughness $\left(R_{\mathrm{a}}\right)$ of less than $0.5 \mathrm{~nm}$. This roughness was not seen to be much affected by the pretreatment methods (i.e. plasma and heat treatments), as concluded from profilometry measurements (Newview 5010, Zygo Corporation, USA).

\section{Sample preparation}

Prior to a given pretreatment, the fused silica hemispheres were first sonicated in ethanol, rinsed in water, immersed in chromosulfuric acid for 30 minutes, and then finally rinsed once again in water. Thereafter, depending on the pretreatment method, samples were either placed in an oven (Thermolyne-47900Furnace) for 4 hours at $1000{ }^{\circ} \mathrm{C}$ and then left to cool-down overnight, or plasma cleaned for 10 minutes using air as carrier gas (Harrick, PDC-32G). Finally, to remove traces of organic contamination, just before experiments, all hemispheres were once again immersed in chromosulfuric acid for 5 minutes and thoroughly rinsed in ultrapure water. Additional details can be found in the ESI. $\dagger$

For the $\mathrm{pH}$ dependent measurements, the $\mathrm{pH}$ was adjusted with either $\mathrm{HCl}$ or $\mathrm{NaOH}$, and solutions were then promptly drawn into gas-tight syringes. Solutions were then transferred into a gas-tight measuring cell, the design of which is described in detail elsewhere. ${ }^{32}$ For performing the measurements of the free silanol in air (see Fig. 3 below), the hemispheres were placed wet in an open cell exposed to a laminar flow of carbonfiltered dry air. SF spectra were then collected during the drying procedure, until the thin wetting film of liquid water evaporated from the surface. The free silanol vibration was then measured without delay at the different polarization combinations. We note that after 20-50 minutes, hydrocarbon contaminants could be detected on the silica surface, together with a measurable decrease in the free silanol vibration intensity at $3750 \mathrm{~cm}^{-1}$. This was not surprising considering the high surface energy of a clean silica surface. Further, we found that drying the hemisphere by forced convection using either filtered dry air or nitrogen, only quickened the rate at which organic contaminants were adsorbed.

Thin wetting films. During the drying procedure when a thin film of water was still wetting the silica substrate, the SF spectra showed a complex interference pattern resulting from the two approaching interfaces (i.e. silica/water and water/air). The sharp free-silanol band at $\sim 3750 \mathrm{~cm}^{-1}$ was absent in the spectra, showing instead the free-OH from water molecules $\left(\sim 3705 \mathrm{~cm}^{-1}\right)$ in the more distant air/water interface, which was typically just a few tens to hundreds of nanometers away, plus the $\nu_{\mathrm{iSiOH}}\left(\sim 3680 \mathrm{~cm}^{-1}\right)$ from the silica/water interface. The interference pattern changed dramatically with the film thickness, as the SF signals from both interfaces could either constructively or destructively interfere. This effect was particularly evident in our SF spectrometer, which has a high resolution and signal to noise ratio. A systematic study of this process will be presented elsewhere. We note, however, that other authors ${ }^{33}$ have neglected interference effects in the interpretation of their results, and we 
believe they have mistakenly assigned the free-OH from water molecules in the thin wetting film to surface silanols.

\section{Sum frequency spectrometer}

Spectra were collected using a femtosecond SF spectrometer and following a normalization procedure described in detail elsewhere. ${ }^{32}$ Briefly, the spectrometer consists of a Ti:Sapphire $\sim 90 \mathrm{fs}, 1 \mathrm{kHz}, 6 \mathrm{~W}$ amplifier (Integra-C, Amplitude Technologies, France), which is partly used to pump a HE-TOPAS (Light Conversion, Lithuania) to generate a broadband $\left(\sim 250 \mathrm{~cm}^{-1}\right)$ tunable IR pulse. Measurements were carried out with a spectral resolution of $<2 \mathrm{~cm}^{-1}$. Angles of incidence for the IR and visible pulses were set to $55.0^{\circ}$ and $70.0^{\circ}$, respectively. Laser energies for the IR and visible beams were set at $\sim 3 \mu \mathrm{J}$ per pulse and $\sim 7 \mu \mathrm{J}$ per pulse, respectively. The non-resonant background from a gold-coated hemisphere was used for normalization. All experiments were repeated on at least three different occasions and using different silica substrates. In all cases, the general spectral features presented here were reproduced, and the effect of the pretreatment methods was found to be reversible. To highlight the differences in the spectral region of interest and following SF community standards, the spectra shown here have not been Fresnel-factor corrected (see ESI $\dagger$ for details of the Fresnel-factor correction procedure and corresponding spectra).

The spectra were fitted by convoluted Lorentzian and Gaussian line shapes of the form presented in eqn (1), which accounts for homogeneous (Lorentzian line shapes) and inhomogeneous broadening (Gaussian line shape), as well as the complex interference between neighbouring bands. ${ }^{34}$

$$
I_{\mathrm{SF}} \propto\left|A_{\mathrm{NR}}^{(2)}+\sum_{v} \int_{-\infty}^{\infty}\left(\frac{-A_{v} \mathrm{e}^{-\left(\omega_{v}{ }^{\prime}-\omega_{v}\right)^{2} / 2 \sigma_{v}{ }^{2}}}{\sqrt{2 \pi \sigma_{v}{ }^{2}}\left(\omega_{\mathrm{IR}}-\omega_{v}{ }^{\prime}+i \Gamma_{v}\right)}\right) \mathrm{d} \omega_{v}{ }^{\prime}\right|^{2}
$$

$A_{\mathrm{NR}}$ refers to the non-resonant contribution to the SF signal, $A_{v}$ to the amplitude or oscillator strength of the $v$ th resonant mode, $\omega_{\mathrm{IR}}$, to the infrared frequency, and $\omega_{v}, \Gamma_{v}$, and $\sigma_{v}$ to the peak position, Lorentzian line width, and Gaussian line width of the $v$ th resonant mode, respectively. As bands in the $\mathrm{OH}$ stretching region are typically dominated by inhomogeneous broadening $\left(\sigma_{v}>\Gamma_{v}\right)$, the $\Gamma_{v}$ was set to $15 \mathrm{~cm}^{-1}$.

\section{Results and discussion}

The starting point of the discussion is the SF spectra of plasma and heat treated fused silica surfaces collected in pure $\mathrm{H}_{2} \mathrm{O}$ under three different polarization combinations (SSP, PPP and SPS; Fig. 1A and B). In addition to the previously identified broad $\mathrm{OH}$ stretching bands, centered at $\sim 3200 \mathrm{~cm}^{-1}$, $\sim 3400 \mathrm{~cm}^{-1}, 15,16,19,21,35$ and $\sim 3500 \mathrm{~cm}^{-1}$ (the latter more clearly resolved in the SPS spectra), ${ }^{21}$ which have all been linked to interfacial water molecules, at higher frequencies, a sharper feature can be observed. This peak centered at $\sim 3680 \mathrm{~cm}^{-1}$ appears more prominently in the heat treated sample, and in contrast to a similar feature observed in the TIR Raman spectra of identical hemispheres, ${ }^{36}$ it originates from accessible surface $\mathrm{OH}$ groups as they can be easily exchanged upon isotopic dilution (Fig. 1C). Thermal treatment, particularly at temperatures above $1000{ }^{\circ} \mathrm{C}$, results in an almost total dehydroxylation of silica surfaces with the interconversion of most silanol groups into siloxane bridges (Si-O-Si). ${ }^{4,6,7,10,37}$ During the heating process, the hydrogenbonded and vicinal silanols are the first to disappear, followed later by the geminals. ${ }^{3,6}$ On the other hand, isolated silanols first increase in numbers, peaking at $\sim 400{ }^{\circ} \mathrm{C}$, before totally dehydroxylating at temperatures above $1100{ }^{\circ} \mathrm{C}^{2,6,10}$ Once the silica substrate is immersed in water the surface rapidly rehydroxylizes, yet only partially. ${ }^{6}$ This effect can be visualized when comparing the SF spectra in Fig. 1A and B. The intensities of the broad water bands at $3200 \mathrm{~cm}^{-1}$ and $\sim 3400 \mathrm{~cm}^{-1}$ in the heat treated sample are approximately $40 \%$ lower than for plasma cleaned silica. Most of the intensity observed in the SF spectra at lower wavenumbers is coupled to water molecules aligned or perturbed by the surface electric field that emanates from deprotonated surface silanols (i.e. silanolate groups). ${ }^{15,21,25}$ The lower intensity in the heat treated sample is then explained by a reduction in the number of silanols that are more easily deprotonated.

The band of interest at higher frequencies follows a different behavior, as in absolute terms it appears not to significantly change between the two pretreatment methods (see Fig. 1A and B). Interferences with neighboring bands, however, as well as a lack of unique fits to the spectra (see below), make difficult a more detailed and reliable analysis. This limitation can be
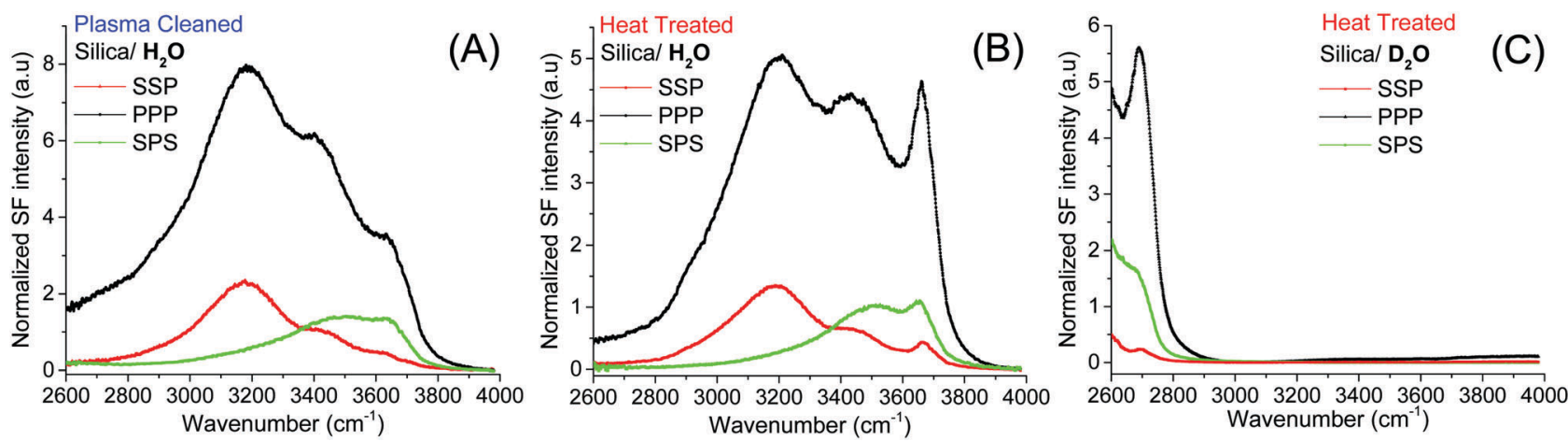

Fig. 1 VSF spectra recorded in the SSP, PPP, and SPS polarization combinations of the (A) plasma, and (B) heat treated fused silica in pure $\mathrm{H}_{2} \mathrm{O}$. (C) VSF spectra of heat treated silica in $\mathrm{D}_{2} \mathrm{O}$. Equivalent Fresnel factor corrected spectra can be found in the ESI. $\dagger$ The absolute intensities between the different spectra can be directly compared. 
largely overcome screening the surface electric field by increasing the ionic strength, as the dominant contribution from the water bands on the red side of the spectra will be significantly diminished. ${ }^{21,35}$ Fig. 2A and B show SF spectra for the two pretreated silica samples in a $10 \mathrm{mM} \mathrm{NaCl}$ solution. When compared to pure $\mathrm{H}_{2} \mathrm{O}$, the intensity of the $\sim 3200 \mathrm{~cm}^{-1}$ peak, but also of that at $\sim 3400 \mathrm{~cm}^{-1}$ are, as expected, much reduced by the screening effect of ions in solution. Still, as for the case of pure water, these latter bands remain less intense in both polarizations (Fig. 2A and B) for the heat treated sample, a fact that is consistent with the above mentioned interpretation of having a smaller population of the more acidic silanols.

On the other hand, the $\sim 3680 \mathrm{~cm}^{-1}$ band that is now more evident, not only shows an opposite trend, being clearly stronger in the heat treated sample in both the SSP and PPP spectra; but also, it transpires to be largely insensitive to the surface electric field as it is not much affected by the increase in the ionic strength. The fact that in all polarization combinations the intensity of the $\sim 3680 \mathrm{~cm}^{-1}$ band is higher for the heat treated sample (see Fig. $2 \mathrm{~A}$ and $\mathrm{B}$ and $\mathrm{ESI} \dagger$ ), is rationalized by a relative increase of the total number of contributing oscillators, and not by a simple change in their average orientation. Due to its high frequency, the band could, in principle, be assigned to either the $\mathrm{OH}$ stretch of water molecules vibrating free from hydrogen bonds ${ }^{38}$ (i.e. next to surface siloxane groups) or to the $\mathrm{OH}$ stretch of specific surface silanol groups (i.e. isolated). As developed below, here we argue in favor of the latter.

\section{Isolated silanols in air}

To determine the relative amounts of silanol groups in both pretreated fused silica substrates, SF spectra were collected on the same surfaces after exposure to a gentle stream of filtered dry air (Fig. 3A and B). As shown in Fig. 3, the spectra are dominated by a narrow peak centered at $\sim 3750 \mathrm{~cm}^{-1}$, which has been unambiguously assigned to the $\mathrm{OH}$ stretch of isolated silanols vibrating in air. ${ }^{3,7,9,39}$ From previous IR studies, ${ }^{4,7,39,40}$ the vibrational signature for hydrogen bonded silanols in air (see Scheme 1) is expected to be broader and red shifted to $\sim 3450-3550 \mathrm{~cm}^{-1}$, while those for vicinal and geminal remain a source of debate, with tentative assignments ranging anywhere between 3500 and $3740 \mathrm{~cm}^{-1}$. Interestingly, in the SF spectra shown in Fig. 3 the population of isolated silanols is clearly higher for the heat-treated sample. Since as discussed above, a larger number of the more acidic silanols (i.e. hydrogen bonded silanols) is formed when plasma cleaning, the direct implication is that isolated silanols are less readily deprotonated. Further, the slightly higher intensities observed at $\sim 3500 \mathrm{~cm}^{-1}$ and $\sim 3700 \mathrm{~cm}^{-1}$ in plasma treated sample (see Fig. 3), are then consistent with the expected relatively larger number of hydrogen bonded silanols, but also probably vicinal and/or geminal. It is worth mentioning, however, that the relatively low contribution of these latter silanol groups to the SF intensity, likely results from the orientation of their $\mathrm{OH}$ groups being aligned, on average, close to the plane of the surface (VSFS is insensitive to such molecular orientations ${ }^{41}$ ).

Accordingly, we assign the $\sim 3680 \mathrm{~cm}^{-1}$ feature to the $\mathrm{OH}$ stretch of isolated silanols vibrating under water $\left(v_{\text {isioH }}\right)$. The red shift of $\sim 70 \mathrm{~cm}^{-1}$ is compatible with the gradual shift observed in silica powders when exposed to a series of gases, including water vapor at low surface coverages. ${ }^{9}$ The advantage here is that VSFS allows detecting such vibrations when silica is in contact with a bulk liquid (Fig. 2). The high frequency of this band indicates that the $\mathrm{OH}$ of these isolated silanols is a relatively strong covalent bond that is not significantly perturbed (i.e. $\sim 70 \mathrm{~cm}^{-1}$ red shift) by hydrogen bonding with adsorbed water molecules. Here, we note that an $\mathrm{OH}$ band with similar characteristics to the $\nu_{\mathrm{iSiOH}}$ has been identified on the surfaces of $\alpha$-Alumina and assigned to a "dangling $(\mathrm{Al})_{n} \mathrm{OH}$ ". ${ }^{42}$ However, in contrast to silica, the frequency of the $(\mathrm{Al})_{n} \mathrm{OH}$ band centred at $\sim 3700 \mathrm{~cm}^{-1}$, remains unchanged whether it is exposed to water or air. ${ }^{42}$

\section{Acid/base behavior of isolated silanols}

Given the assignment of isolated silanols under water, one of the first properties to explore is its $\mathrm{pH}$ behavior and the
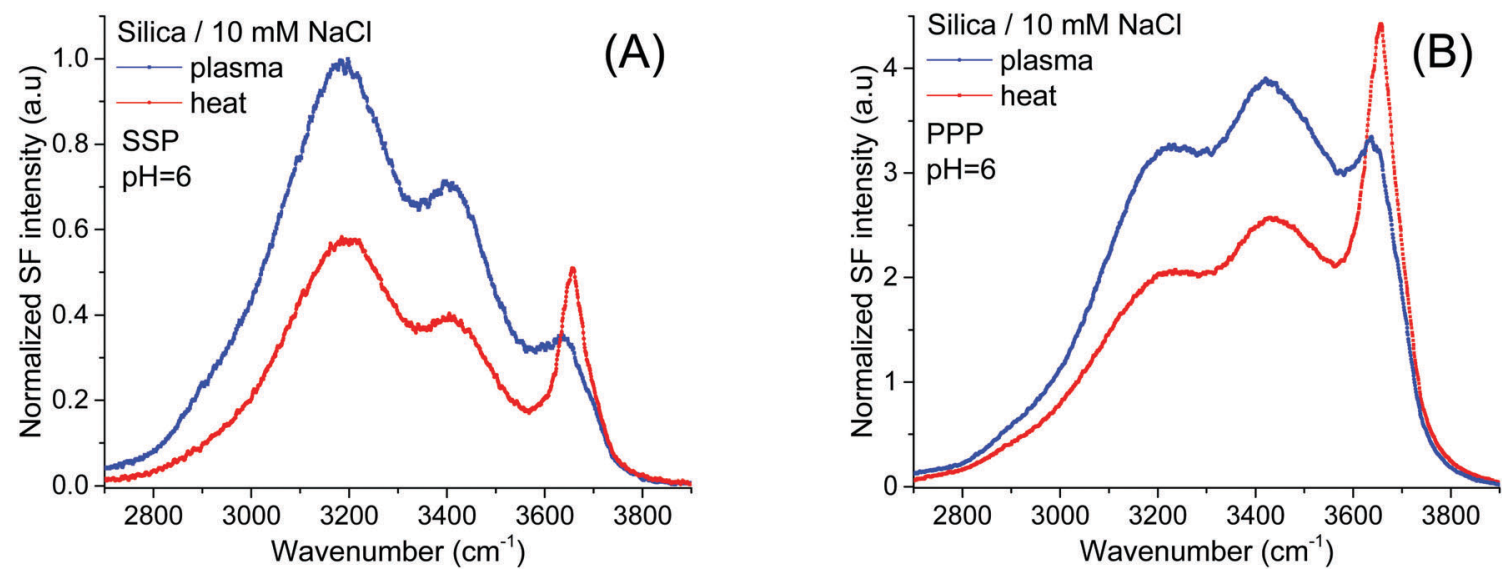

Fig. 2 VSF spectra recorded in the (A) SSP, and (B) PPP polarization combinations of plasma and heat treated fused silica in contact with a $10 \mathrm{mM}$ NaCl solution. Equivalent Fresnel factor corrected spectra can be found in the ESI. $\dagger$ The absolute intensities between the different spectra can be directly compared with those of Fig. 1 and 4. 

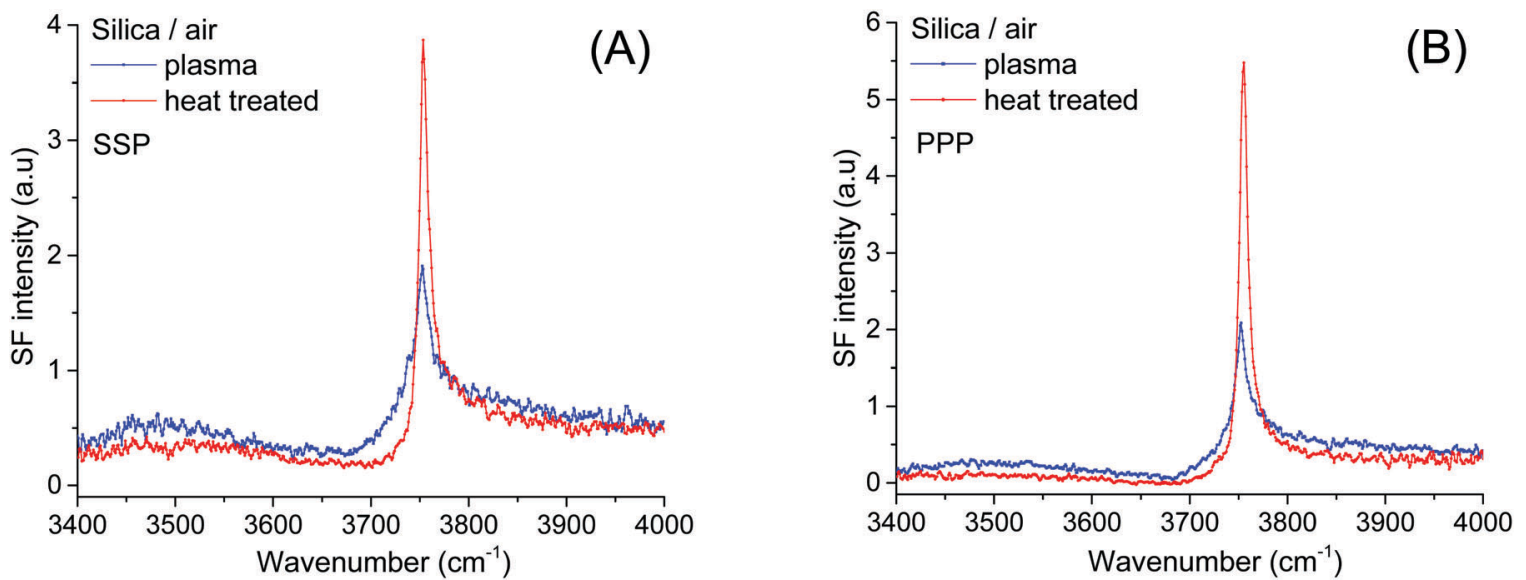

Fig. 3 VSF spectra recorded in the (A) SSP, and (B) PPP polarization combinations of plasma and heat treated fused silica dried in a gentle stream of dry filtered air. The fact that the $\mathrm{SiO}-\mathrm{H}$ stretch of isolated silanols remains stronger in both polarization combinations for the heat treated sample implies more contributing oscillators rather than a change in their orientation.

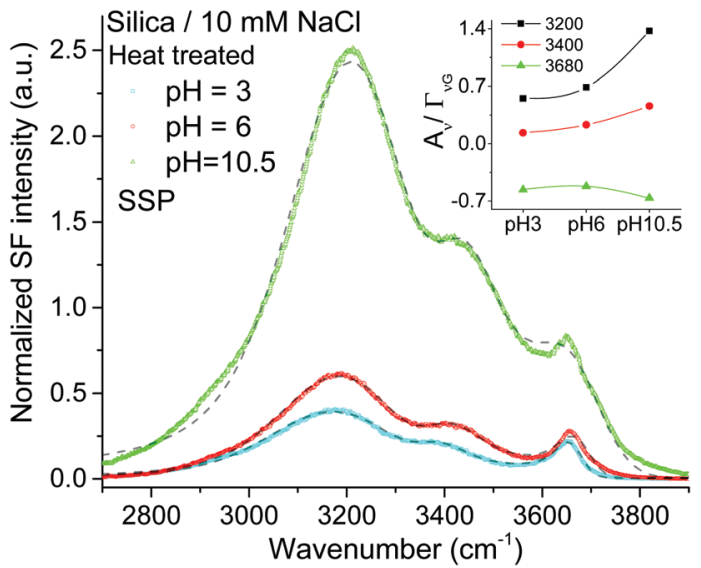

Fig. 4 VSF spectra recorded in the SSP polarization combination of a heat treated fused silica hemisphere in contact with $10 \mathrm{mM} \mathrm{NaCl}$ solution at three different pHs (PPP spectra can be found in the ESI†). The shortsegmented lines are the fitted curves to the experimental data. Data were collected in the following sequence: $\mathrm{pH} 6, \mathrm{pH} 3$, and finally $\mathrm{pH}$ 10.5. The absolute intensities between the different spectra can be directly compared with those of Fig. 1 and 2. Equivalent Fresnel factor corrected spectra can be found in the ESI. $\dagger$ Inset: Fitted amplitude of the bands at $\sim 3200 \mathrm{~cm}^{-1}, \sim 3400 \mathrm{~cm}^{-1}$ and $\sim 3680 \mathrm{~cm}^{-1}$ (see ESI $\dagger$ for details).

determination of its eventual $\mathrm{p} K_{\mathrm{a}}$. Fig. 4 shows the SF spectra of a heat treated silica sample at three different pHs using $10 \mathrm{mM}$ sodium chloride as background electrolyte (equivalent PPP spectra can be found in the ESI $\dagger$ ). As expected, the contribution from the broad water bands at $3200 \mathrm{~cm}^{-1}$ and $\sim 3400 \mathrm{~cm}^{-1}$ increases with solution $\mathrm{pH}$, as the surface charge increases with the partial deprotonation of surface silanol groups. ${ }^{15,16}$ Interestingly, the $\nu_{\mathrm{iSioH}}$ remains visible even at $\mathrm{pH}=10.5$, which implies that the isolated silanols do not easily deprotonate. A more quantitative description can be obtained by fitting the spectra to the convoluted Lorentzian and Gaussian lineshapes described in more detail in the Experimental section. The fitted amplitudes for three resonant contributions as a function of $\mathrm{pH}$ are summarized in the inset of Fig. 4 (see ESI $\dagger$ for additional details).
The results show that while the amplitudes for the $3200 \mathrm{~cm}^{-1}$ and $\sim 3400 \mathrm{~cm}^{-1}$ bands increase with $\mathrm{pH}$, the one linked to the isolated silanols not only remains approximately constant, but also displays an opposite sign. Due to the coherent nature of the SF process, information is carried both in the magnitude and phase of the SF signal. ${ }^{35}$ Consistent with the proposed assignment, the reversed sign indicates that the $\mathrm{OH}$ groups of isolated silanols have an opposite net polar orientation from those in water molecules aligned by the negative surface electric field (i.e. water hydrogens are oriented on average towards the surface, ${ }^{22}$ while those in silanols point away from the surface). Had the surface been positively charged, all three modes would have had the same phase (i.e. sign), as the $\mathrm{OH}$ groups from both the interfacial water molecules and isolated silanols would be, on average, oriented away from the surface. As shown in Fig. 5, this is indeed the case when the positively charged surfactant CTAB adsorbs to silica at a concentration above its critical micellar concentration (see ESI $\dagger$ for fitting parameters). ${ }^{43}$ Interestingly, due to interference with the water neighboring band, the $\nu_{\mathrm{iSiOH}}$ is now observed as a dip, instead of a peak in the spectrum (see Fig. 5). Clearly, this situation illustrates how the $v_{\mathrm{iSiOH}}$ can be used as an internal reference for determining the absolute polar orientation of water molecules in the interfacial region. Moreover, the fact that the $\nu_{\mathrm{iSiOH}}$ band remains visible for the CTAB case, also helps discarding the possibility of associating the $\sim 3680 \mathrm{~cm}^{-1}$ band to water molecules weakly interacting with the silica substrate (see above), as these water molecules would have necessarily been displaced by the adsorbing surfactant.

Returning to Fig. 4, the lack of significant changes in the fitted amplitudes for $\nu_{\mathrm{iSiOH}}$ with increasing $\mathrm{pH}$, indicates that the $\mathrm{p} K_{\mathrm{a}}$ of isolated silanols is higher than 10.5. Studies using higher ionic strength solutions to be presented elsewhere situate the $\mathrm{p} K_{\mathrm{a}}$ above 11.5. It is worth noting, however, that the experimentally accessible range is limited due to the dissolution of silica at high $\mathrm{pH}$, which is already expected at $\mathrm{pH}$ above 11 for amorphous precipitated silica, ${ }^{2}$ and is indeed observed in our substrates when exceeding $\mathrm{pH} 12$ at room temperature. In 


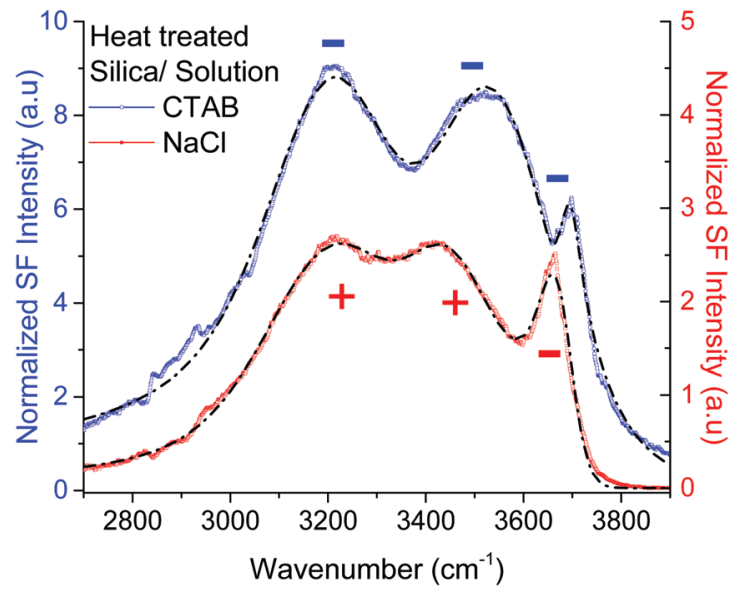

Fig. 5 VSF spectra recorded in the PPP polarization combination of a heat treated fused silica hemisphere in contact with a $2 \mathrm{mM}$ CTAB solution (positively charged surface), and a $10 \mathrm{mM} \mathrm{NaCl}$ solution (negatively charged surface). The short-segmented lines are the fitted curves to the experimental data where the $\mathrm{CH}$ modes have been neglected (see ESI $\dagger$ for details of the fitting parameters). For the positively charged surface (CTAB) the amplitudes of the water bands at $\sim 3200 \mathrm{~cm}^{-1}, \sim 3400 \mathrm{~cm}^{-1}$ have the same sign (i.e. minus) as for $\nu_{\mathrm{iSiOH}}$ at $\sim 3680 \mathrm{~cm}^{-1}$, while for the negatively charged surface $(10 \mathrm{mM} \mathrm{NaCl})$, the water bands have opposite sign since the water $\mathrm{OHs}$ are now oriented, on average, towards the silica substrate. Note that because of interference, the $\nu_{\mathrm{isiOH}}$ appears as a dip instead of peak in the CTAB spectrum.

line with these experimental findings, recent molecular dynamic simulations $\mathrm{s}^{27,29}$ have estimated the $\mathrm{p} K_{\mathrm{a}}$ of isolated silanols to be higher than those for hydrogen bonded and geminal silanols, with calculated values ranging between 8.9 and 11.2, although in the presence of specific monovalent ions the $\mathrm{p} K_{\mathrm{a}}$ have been estimated to shift several units upwards. ${ }^{44}$

In context of the results presented here, and recalling that a large majority $(>75 \%)$ of surface silanols do not dissociate even at $\mathrm{pH}$ as high as $10,{ }^{23,30}$ the silanols sites inferred from second harmonic data having a $\mathrm{p} K_{\mathrm{a}} \sim 4.5$ and $\sim 8.5,{ }^{17}$ are clearly not associated with isolated $\mathrm{Si}-\mathrm{OHs}$, but instead with those having closely neighboring silanols: vicinal, geminal, and/or hydrogenbonded. Further, the relative populations of the different silanol sites, will evidently depend on the pretreatment history of the silica substrate, a fact that may partly explain the differences observed in more recent $\mathrm{SH}$ studies. ${ }^{23}$

\section{Conclusions}

In summary, we have used VSFS to identify the SiO-H stretch of isolated $\mathrm{Q}^{3}$ surface silanols in contact with bulk water. Although relatively narrow, the feature $\left(\nu_{\mathrm{iSiOH}}\right)$ is broader and red-shifted by $\sim 70 \mathrm{~cm}^{-1}$ when compared to its free vibration in air. In addition, we show that depending on the silica pretreatment, the relative population of the different types of silanols under water can be modified. Preheating the fused silica sample to $1000{ }^{\circ} \mathrm{C}$ results in a larger number of isolated silanols when compared to plasma cleaning using air as carrier gas, while the opposite trend is observed for the more acidic Si-OHs (i.e. hydrogen bonded and/or geminal). This behavior was found to be reversible. Moreover, $\mathrm{pH}$ dependent studies show that the $\mathrm{p} K_{\mathrm{a}}$ of the isolated silanols is higher than 10.5. Finally, the identification of the isolated silanol band opens new opportunities to further understand the mechanism of adsorption of various molecules, including specific ion interactions, ${ }^{44-47}$ on the surface of this commonly used material.

\section{Acknowledgements}

This work was financially supported by the Swedish Foundation for Strategic Research (SSF-FFL-5 program) and the Swedish Research Council (VR). The authors thank Marie-Pierre Gaigeot and Julianne Gibbs-Davis for insightful discussions. Jonathan Liljeblad is acknowledged for developing the routine used for fitting the SF spectra.

\section{References}

1 G. Schubert, Treatise on Geophysics, Elsevier Science, 2015.

2 R. K. Iler, The chemistry of silica: solubility, polymerization, colloid and surface properties, and biochemistry, Wiley, 1979.

3 A. Rimola, D. Costa, M. Sodupe, J. F. Lambert and P. Ugliengo, Chem. Rev., 2013, 113, 4216-4313.

4 H. E. Bergna and W. O. Roberts, Colloidal Silica: Fundamentals and Applications, CRC Press, 2005.

5 W. Tan, K. Wang, X. He, X. J. Zhao, T. Drake, L. Wang and R. P. Bagwe, Med. Res. Rev., 2004, 24, 621-638.

6 L. T. Zhuravlev, Colloids Surf., A, 2000, 173, 1-38.

7 E. Papirer, Adsorption on Silica Surfaces, Taylor \& Francis, 2000.

8 G. E. Maciel and D. W. Sindorf, J. Am. Chem. Soc., 1980, 102, 7606-7607.

9 R. S. McDonald, J. Am. Chem. Soc., 1957, 79, 850-854.

10 V. I. Lygin, Russ. J. Gen. Chem., 2001, 71, 1368-1372.

11 L. T. Zhuravlev, Langmuir, 1987, 3, 316-318.

12 A. G. Lambert, P. B. Davies and D. J. Neivandt, Appl. Spectrosc. Rev., 2005, 40, 103-145.

13 C. D. Bain, J. Chem. Soc., Faraday Trans., 1995, 91, 1281-1296.

14 K. B. Eisenthal, Chem. Rev., 2006, 106, 1462-1477.

15 Q. Du, E. Freysz and Y. R. Shen, Phys. Rev. Lett., 1994, 72, 238-241.

16 V. Ostroverkhov, G. A. Waychunas and Y. R. Shen, Chem. Phys. Lett., 2004, 386, 144-148.

17 S. Ong, X. Zhao and K. B. Eisenthal, Chem. Phys. Lett., 1992, 191, 327-335.

18 S. Dewan, M. S. Yeganeh and E. Borguet, J. Phys. Chem. Lett., 2013, 4, 1977-1982.

19 O. Isaienko, S. Nihonyanagi, D. Sil and E. Borguet, J. Phys. Chem. Lett., 2013, 4, 531-535.

20 P. A. Covert, K. C. Jena and D. K. Hore, J. Phys. Chem. Lett., 2014, 5, 143-148.

21 K. C. Jena and D. K. Hore, J. Phys. Chem. C, 2009, 113, 15364-15372. 
22 A. Myalitsin, S.-H. Urashima, S. Nihonyanagi, S. Yamaguchi and T. Tahara, J. Phys. Chem. C, 2016, 120, 9357-9363.

23 A. M. Darlington and J. M. Gibbs-Davis, J. Phys. Chem. C, 2015, 119, 16560-16567.

24 D. Lis, E. H. G. Backus, J. Hunger, S. H. Parekh and M. Bonn, Science, 2014, 344, 1138-1142.

25 V. Ostroverkhov, G. A. Waychunas and Y. R. Shen, Phys. Rev. Lett., 2005, 94, 046102.

26 M. Sovago, R. Kramer Campen, H. J. Bakker and M. Bonn, Chem. Phys. Lett., 2009, 470, 7-12.

27 K. Leung, I. M. B. Nielsen and L. Criscenti, Geochim. Cosmochim. Acta, 2010, 74, A584-A584.

28 M. Sulpizi, M. P. Gaigeot and M. Sprik, J. Chem. Theory Comput., 2012, 8, 1037-1047.

29 M. Pfeiffer-Laplaud, D. Costa, F. Tielens, M.-P. Gaigeot and M. Sulpizi, J. Phys. Chem. C, 2015, 119, 27354-27362.

30 M. A. Brown, A. Goel and Z. Abbas, Angew. Chem., Int. Ed., 2016, 55, 3790-3794.

31 T. Lagstrom, T. A. Gmur, L. Quaroni, A. Goel and M. A. Brown, Langmuir, 2015, 31, 3621-3626.

32 J. F. D. Liljeblad and E. Tyrode, J. Phys. Chem. C, 2012, 116, 22893-22903.

33 O. Isaienko and E. Borguet, Langmuir, 2013, 29, 7885-7895.

34 C. D. Bain, P. B. Davies, T. H. Ong, R. N. Ward and M. A. Brown, Langmuir, 1991, 7, 1563-1566.
35 Y. R. Shen and V. Ostroverkhov, Chem. Rev., 2006, 106, 1140-1154.

36 J. F. D. Liljeblad, I. Furo and E. C. Tyrode, Phys. Chem. Chem. Phys., 2017, 19, 305-317.

37 J. P. Gallas, J. C. Lavalley, A. Burneau and O. Barres, Langmuir, 1991, 7, 1235-1240.

38 Q. Du, E. Freysz and Y. R. Shen, Science, 1994, 264, 826-828.

39 B. A. Morrow and A. J. McFarlan, J. Phys. Chem., 1992, 96, 1395-1400.

40 S. P. Zhdanov, L. S. Kosheleva and T. I. Titova, Langmuir, 1987, 3, 960-967.

41 R. Lu, W. Gan, B.-H. Wu, Z. Zhang, Y. Guo and H.-F. Wang, J. Phys. Chem. B, 2005, 109, 14118-14129.

42 L. Zhang, C. Tian, G. A. Waychunas and Y. R. Shen, J. Am. Chem. Soc., 2008, 130, 7686-7694.

43 E. Tyrode, M. W. Rutland and C. D. Bain, J. Am. Chem. Soc., 2008, 130, 17434-17445.

44 M. Pfeiffer-Laplaud, M.-P. Gaigeot and M. Sulpizi, J. Phys. Lett., 2016, 7, 3229-3234.

45 S. Dewan, V. Carnevale, A. Bankura, A. Eftekhari-Bafrooei, G. Fiorin, M. L. Klein and E. Borguet, Langmuir, 2014, 30, 8056-8065.

46 M. S. Azam, C. N. Weeraman and J. M. Gibbs-Davis, J. Phys. Lett., 2012, 3, 1269-1274.

47 T. A. Gmür, A. Goel and M. A. Brown, J. Phys. Chem. C, 2016, 120, 16617-16625. 